\title{
A mobile one-lead ECG device incorporated in a symptom- driven remote arrhythmia monitoring program. The first 5,982 Hartwacht ECGs
}

\author{
J. L. Selder · L. Breukel · S. Blok · A. C. van Rossum • I. I. Tulevski · C. P. Allaart
}

Published online: 6 December 2018

(c) The Author(s) 2018

\begin{abstract}
Background In recent years many mobile devices able to record health-related data in ambulatory patients have emerged. However, well-organised programs to incorporate these devices are sparse. Hartwacht Arrhythmia (HA) is such a program, focusing on remote arrhythmia detection using the AliveCor Kardia Mobile (KM) and its algorithm.

Objectives The aim of this study was to assess the benefit of the KM device and its algorithm in detecting cardiac arrhythmias in a real-world cohort of ambulatory patients.

Methods All KM ECGs recorded in the HA program between January 2017 and March 2018 were included. Classification by the KM algorithm was compared with that of the Hartwacht team led by a cardiologist. Statistical analyses were performed with respect to detection of sinus rhythm (SR), atrial fibrillation (AF) and other arrhythmias.

Results 5,982 KM ECGs were received from 233 patients (mean age 58 years, 52\% male). The KM algorithm categorised $59 \%$ as SR, $22 \%$ as possible AF, $17 \%$ as unclassified and $2 \%$ as unreadable. According to the Hartwacht team, 498 (8\%) ECGs were uninterpretable. Negative predictive value for detection of AF was $98 \%$. However, positive predictive value as well as detection of other arrhythmias was poor. In $81 \%$ of
\end{abstract}

J. L. Selder $(\bowtie) \cdot$ A. C. van Rossum · C. P. Allaart

Amsterdam UMC, location VUMC, Amsterdam, Netherlands j.selder@vumc.nl

L. Breukel

Onze Lieve Vrouwe Hospital, Amsterdam, Netherlands

S. Blok · I. I. Tulevski

Cardiology Center Netherlands, Amsterdam, Netherlands the unclassified ECGs, the Hartwacht team was able to provide a diagnosis.

Conclusions This study reports on the first symptomdriven remote arrhythmia monitoring program in the Netherlands. Less than $10 \%$ of the ECGs were uninterpretable. However, the current performance of the KM algorithm makes the device inadequate as a standalone application, supporting the need for manual ECG analysis in HA and similar programs.

Keywords eHealth - AliveCor - Kardia - Hartwacht · remote monitoring $\cdot$ ECG $\cdot$ arrhythmia

\section{Background}

The healthcare system is a dynamic environment which evolves due to public demand and technological advancement. Currently, within the Netherlands, the system is changing to a state where patients are more responsible for and more in control of their own

\section{What's new}

- The AliveCor Kardia Mobile provides a patient-initiated 30-second one-lead ECG of diagnostic quality in ambulatory arrhythmia patients.

- For incorporation of a one-lead mobile ECG device in a regular healthcare setting, a dedicated arrhythmia program is of additional value as long as algorithms are not reliable enough.

- The future of remote monitoring for arrhythmias depends on custom-tailored algorithms. Refinement of detection of regular sinus rhythm in a way that it obviates the need for manual assessment of this category will reduce workload significantly 


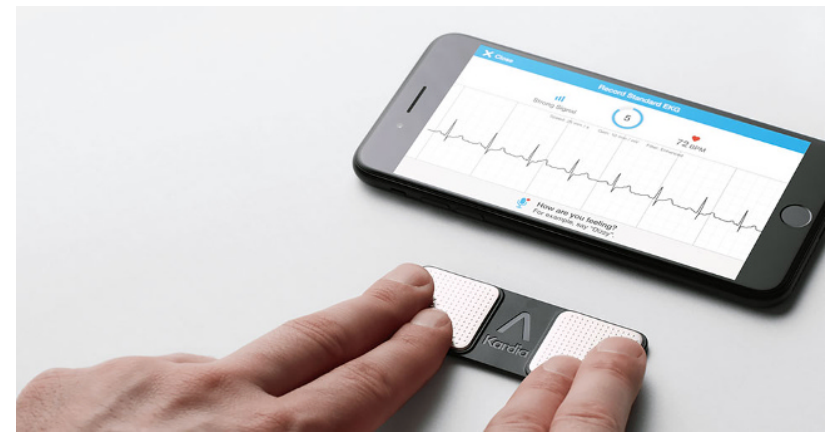

Fig. 1 AliveCor Kardia Mobile device. (Downloaded with permission from www.Alivecor.com)

health, partly by the introduction of mobile health. Many devices and applications have been developed to provide patients with an opportunity to record specific health-related data outside the hospital, and heart rhythm has been an important area of interest in this. These devices are mostly targeted towards the general public and only few have been integrated in the healthcare system through a well-organised program with due regard for practicality, safety and privacy. An example of a well-incorporated device is the AliveCor Kardia Mobile (KM), a handheld ECG device, which is being used by the Hartwacht Arrhythmia (HA) program.

KM is a small ECG device, manufactured by AliveCor, which works by placing one finger of each hand on the electrodes (Fig. 1). A one-lead 30-second ECG (lead I) is recorded and transferred to a connected smartphone through a wireless communication protocol using ultrasonic audio. The ECG is simultaneously detected and locally analysed on the smartphone by the KM algorithm, which will classify it as normal sinus rhythm, possible AF, unclassified or unreadable. Both the ECG and its classification can then be sent on or emailed for further assessment.

The KM device and its algorithm have been assessed and validated in several studies. Sensitivity and specificity varied between $55-100 \%$ and $84-99 \%$ respectively, depending on the patient population and reference technique [1-6].

However, none of these studies have taken place in a real-life outpatient clinic setting. Hartwacht Arrhythmia, a remote monitoring program for heart rhythms in the Netherlands, initiated by Cardiologie Centra Nederland (CCN), provides an opportunity to evaluate the added value of the KM device incorporated in medical care. The HA program combines the KM with the expertise of a dedicated medical team led by a cardiologist for analysis of these ECGs, and thereby provides the opportunity to assess the accuracy of the KM algorithm in a real-life outpatient clinic setting as well as give insight as to the added benefit of this mobile device in the healthcare system. The aim of this study was to assess the benefit of the KM device in detecting cardiac arrhythmias in a realworld cohort of ambulatory patients, by analysing the results of the KM incorporated in the HA program focusing on the accuracy of the KM algorithm compared to KM interpretation of the Hartwacht team.

\section{Methods}

\section{Study population}

The study population consisted of all HA patients who submitted a KM ECG from the start of the program in January 2017 until March 2018. The Hartwacht program is available to patients of CCN, a private outpatient cardiology clinic. Typically, patients presenting with paroxysmal AF, palpitations of unknown origin or near-collapse were selected by the cardiologists of this clinic to participate in the Hartwacht program, although indications for inclusion in the program were left at the discretion of the physician.

\section{Hartwacht}

After inclusion in the HA program, participants received the KM device at home, downloaded the Kardia smartphone application and were instructed on its use by the Hartwacht team. Whenever participants experienced palpitations or related complaints, they were encouraged to record an ECG with the KM device, after which the ECG and its classification by the algorithm were automatically transferred to the patient's electronic patient record. There was no limit to the number of ECGs that could be recorded. ECGs

Table 1 Patient characteristics

\begin{tabular}{|c|c|}
\hline & $N=233$ \\
\hline \multicolumn{2}{|l|}{ demographics } \\
\hline age & $58.4( \pm 14)$ \\
\hline male & $120(52 \%)$ \\
\hline \multicolumn{2}{|l|}{ registered diagnoses } \\
\hline atrial fibrillation/flutter & $127(55 \%)$ \\
\hline other supraventricular tachycardia & $64(28 \%)$ \\
\hline ventricular tachycardia & $24(10 \%)$ \\
\hline impulse and conduction disorder & $10(4 \%)$ \\
\hline stable coronary artery disease & $27(12 \%)$ \\
\hline acute coronary syndrome & $3(1 \%)$ \\
\hline valvular heart disease & $21(9 \%)$ \\
\hline chronic heart failure & $5(2 \%)$ \\
\hline hypertension & $62(27 \%)$ \\
\hline anti-arrhythmic medication & $77(33 \%)$ \\
\hline amiodarone & $2(1 \%)$ \\
\hline beta-blocker & $17(7 \%)$ \\
\hline calcium channel blocker (verapamil, diltiazem) & $23(10 \%)$ \\
\hline flecainide & $14(6 \%)$ \\
\hline digoxin & $14(6 \%)$ \\
\hline disopyramide & $6(3 \%)$ \\
\hline sotalol & $1(0 \%)$ \\
\hline
\end{tabular}


were assessed by the Hartwacht team, consisting of a supervising cardiologist (0.05 FTE), a specialised cardiology nurse (1.0 FTE) and a doctor's assistant (0.02 FTE), working on weekdays from $08.00 \mathrm{hrs}$ to 17.00 hrs. Furthermore, a cardiologist who could directly access all Hartwacht ECGs was available 24/7 for emergency purposes. Patients received feedback from the Hartwacht team within one working day by phone or email.

\section{Data acquisition and analysis}

Anonymised data were obtained from Hartwacht for analysis. Variables included in the analyses were patient characteristics, number of ECGs per patient per month and time of day that ECGs were received, classification of the ECG by the KM algorithm and results of assessment by the Hartwacht team. The KM algorithm classifies each ECG as one of four categories: (a) normal sinus rhythm, (b) possible AF, (c) unclassified or (d) unreadable. Assessment by the Hartwacht team resulted in classification as (a) sinus rhythm, with or without premature atrial contractions (PACs) and/or premature ventricular contractions PVCs, (b) atrial fibrillation, (c) other arrhythmias (including wide and narrow complex tachycardias and complete atrioventricular block) and (d) unin- terpretable. Continuous variables were presented as mean \pm standard deviation, categorical variables as frequencies. Sensitivity, specificity, positive predictive value (PPV), negative predictive value (NPV) and accuracy were calculated from the KM interpretation, with the Hartwacht team interpretation as reference standard. KM ECGs categorised as unclassified or unreadable were included in the sensitivity/specificity calculation when the Hartwacht team was able to provide a diagnosis. For example: An ECG interpreted by the KM algorithm as unclassified and subsequently by the Hartwacht team as atrial fibrillation deemed false negative for $\mathrm{AF}$.

\section{Results}

A total of 233 participants in Hartwacht were included in the study. Patient characteristics are shown in Tab. 1. Seven patients $(3 \%)$ exited the program, mostly because they never made ECGs. During the study period 5,982 KM ECGs were received, with a median of 28 ECGs per patient per year (Fig. 2a). Of these, the KM algorithm categorised 3,548 (59\%) as normal sinus rhythm, $1,301(22 \%)$ as possible atrial fibrillation, 1,033 (17\%) as unclassified and $100(2 \%)$ as unreadable (Fig. 3b). Analysis by the Hartwacht team resulted in 4,235 ECGs classified as sinus rhythm, of
Fig. 2 Number of KM ECGs received per patient per month and timing of received KM ECGs

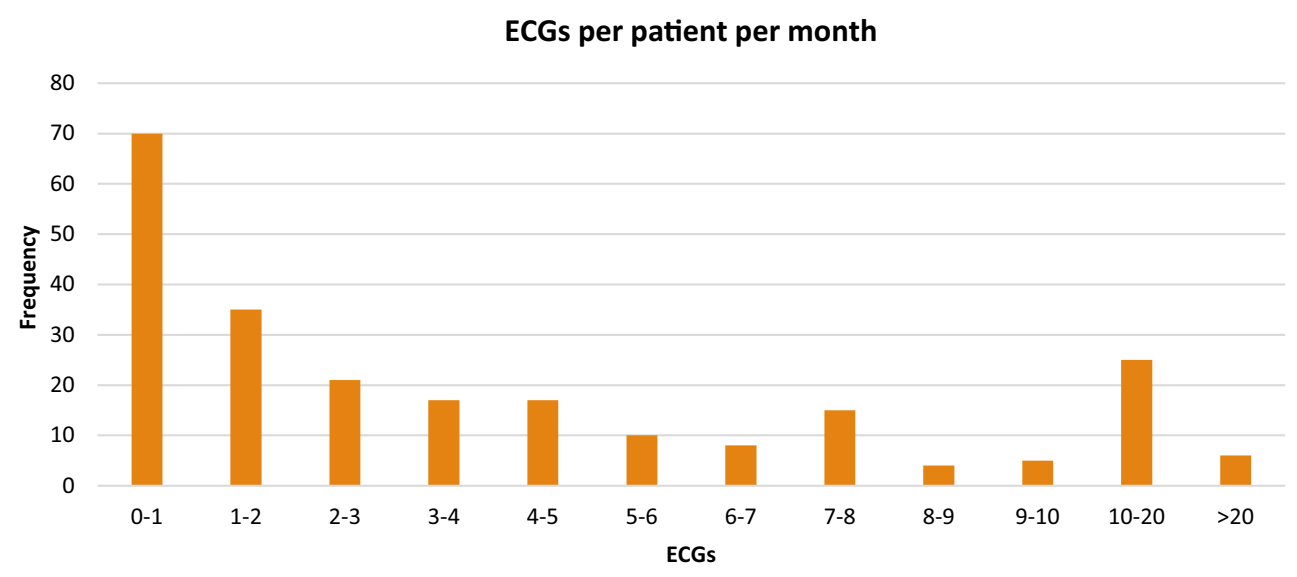

Time ECGs received

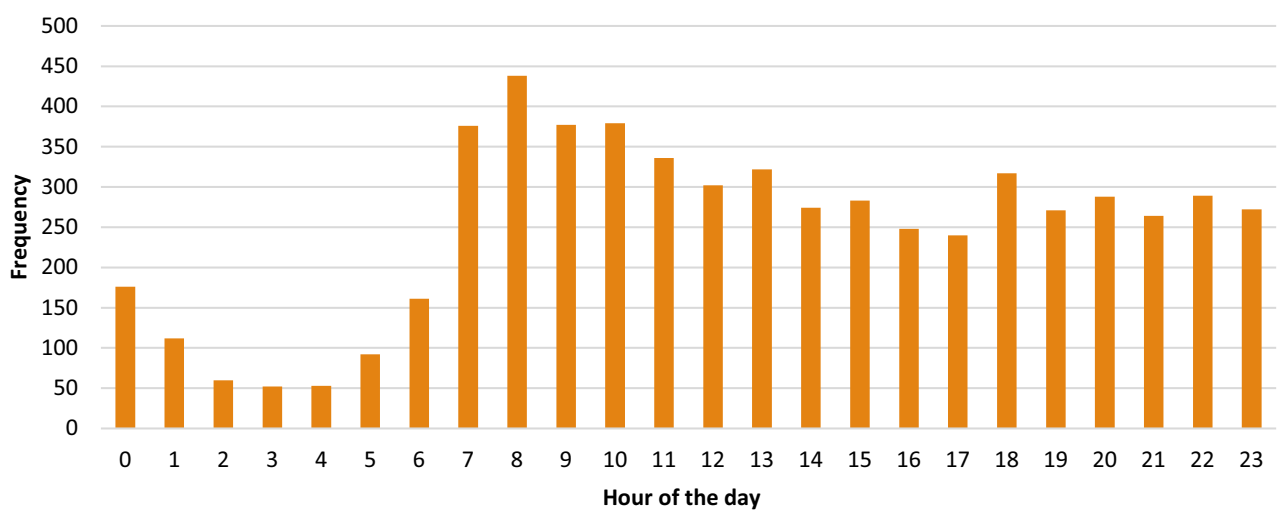


which 476 (8\%) showed ectopy whereas 3,759 (63\%) did not, 1,135 (19\%) ECGs showed atrial fibrillation, $114(2 \%)$ showed other arrhythmias and the remaining 498 were uninterpretable (8\%) (Fig. 3a). Most ECGs were recorded between 07.00 and $11.00 \mathrm{hrs}$. A total of 3,023 ECGs (51\%) were received outside office hours (17.00-08.00 hrs). Distribution of arrhythmias between classification groups in these ECGs did not differ from those received during office hours. Typical ECGs from every category are shown in Fig. 4.

Classification of the ECGs by the KM algorithm and diagnosis of the Hartwacht team differed significantly (Tab. 2). When the ECG was classified as sinus rhythm by the KM algorithm, the Hartwacht team agreed in $96 \%$ (90\% without ectopy, $6 \%$ with ectopy); $4 \%$ were deemed uninterpretable and AF was diagnosed in $<1 \%$. When possible AF was detected by the KM algorithm, the Hartwacht assessment confirmed AF in $80 \%$ of cases. The remainder were diagnosed as sinus rhythm with or without ectopy $(8 \%$ and $4 \%$, respectively), other arrhythmias (1\%) or uninterpretable (7\%). From the ECGs that were classified by the KM algorithm as unclassifiable, the Hartwacht was able to provide a diagnosis in $81 \%$ of cases with $64 \%$ sinus rhythm (13\% with ectopy), $8 \%$ atrial fibrillation, and $10 \%$ other diagnoses (including broad and small complex tachycardia and complete AV block). Even in the category unreadable, $29 \%$ of the ECGs could be interpreted by the Hartwacht team (Tab. 2).

\section{Ectopy and other arrhythmias}

Irregular rhythms other than $\mathrm{AF}$ pose a challenge to the algorithm, which is illustrated by the distribution of ECGs diagnosed as sinus rhythm with ectopy over the KM classes. KM ECGs diagnosed by the cardiologist as sinus rhythm with PACs (5\%) were classified by the KM algorithm as sinus rhythm (47\%), atrial fibrillation $(26 \%)$ or unclassified $(27 \%)$ (Fig. 3c). ECGs with sinus rhythm and PVCs were similarly interpreted (Fig. 3d). In addition to sinus rhythm and atrial fibrillation, a small portion of ECGs (2\%) showed other arrhythmias. For details see Tab. 2. There were several remarkable ECGs, shown in Fig. 4.

\section{Sensitivity and specificity}

Using the assessment of the Hartwacht team as reference standard, the reliability of the different KM classifications in terms of sensitivity, specificity, PPV, NPV and accuracy, was determined (Fig. 5). For diagnosing AF these were $0.92,0.95,0.80,0.98$ and 0.94 respec-
Fig. 3 Interpretation of 5,982 KM ECGs (SR sinus rhythm, $A F$ atrial fibrillation, SCT small complex tachycardia, $P A C$ premature atrial complex, PVC premature ventricular complex)
Interpretation Cardiologist

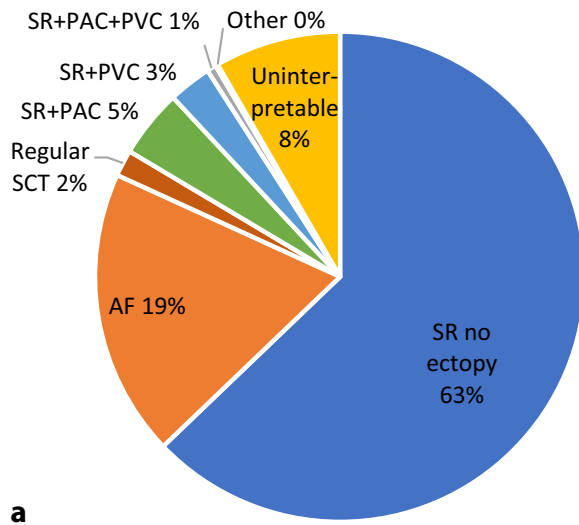

Interpretation SR+PAC by Kardia

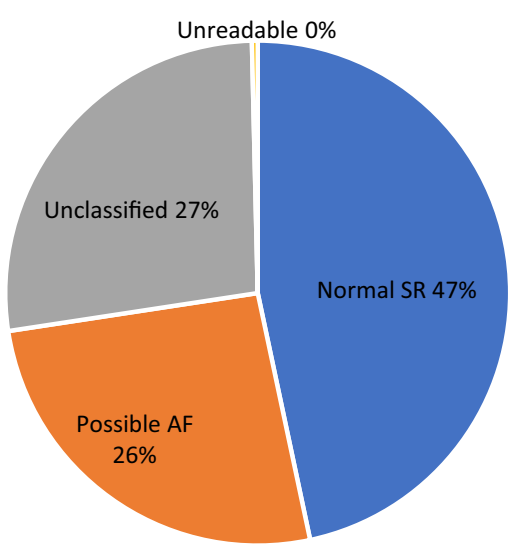

Interpretation Kardia

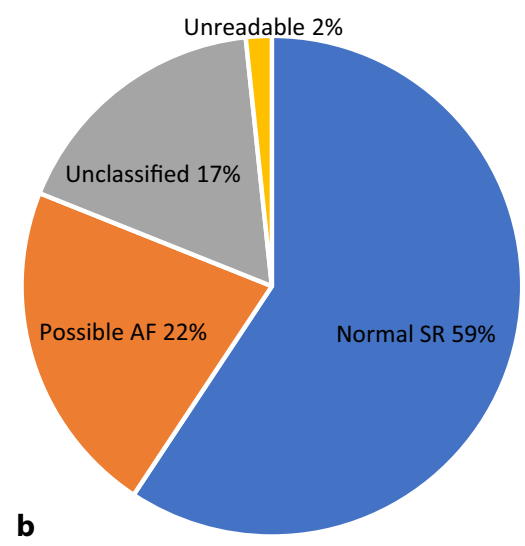

Interpretation SR+PVC by Kardia

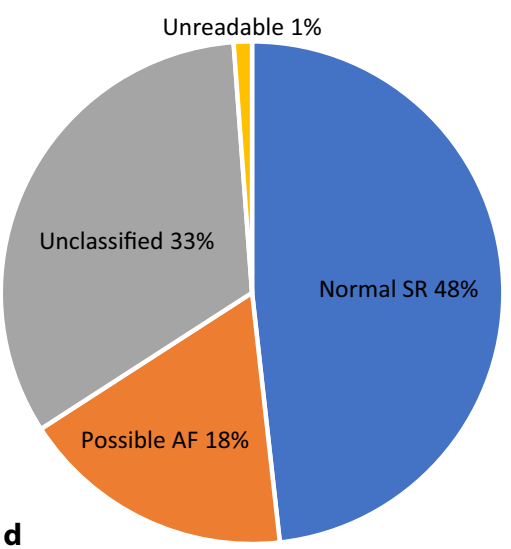


Table 2 Interpretation of the ECGs by the KM algorithm (bold) and subsequent interpretation by the cardiologist (normal)

\begin{tabular}{|c|c|}
\hline normal sinus rhythm (KM) & $3,548(59 \%)$ \\
\hline atrial fibrillation & $11(<1 \%)$ \\
\hline sinus rhythm & $3,394(96 \%)$ \\
\hline - without ectopy & $3,177(90 \%)$ \\
\hline - with PACs & $126(4 \%)$ \\
\hline - with PVCs & $82(2 \%)$ \\
\hline - with PACs and PVCs & $9(<1 \%)$ \\
\hline other arrhythmias & $2(<1 \%)$ \\
\hline - regular small complex tachycardia & $2(<1 \%)$ \\
\hline uninterpretable & $141(4 \%)$ \\
\hline possible atrial fibrillation (KM) & $1,301(22 \%)$ \\
\hline atrial fibrillation & $1,042(80 \%)$ \\
\hline sinus rhythm & $162(12 \%)$ \\
\hline - without ectopy & $49(4 \%)$ \\
\hline - with PACs & $70(5 \%)$ \\
\hline - with PVCs & $30(2 \%)$ \\
\hline - with PACs and PVCs & $13(1 \%)$ \\
\hline other arrhythmias & $10(1 \%)$ \\
\hline - regular small complex tachycardia & $8(1 \%)$ \\
\hline - regular broad complex tachycardia & $1(<1 \%)$ \\
\hline - complete AV block & $1(<1 \%)$ \\
\hline uninterpretable & $87(7 \%)$ \\
\hline unclassified (KM) & $1,033(17 \%)$ \\
\hline atrial fibrillation & $78(8 \%)$ \\
\hline sinus rhythm & $658(64 \%)$ \\
\hline - without ectopy & $515(50 \%)$ \\
\hline - with PACs & $73(7 \%)$ \\
\hline - with PVCs & $56(5 \%)$ \\
\hline - with PACs and PVCs & $14(1 \%)$ \\
\hline other arrhythmias & $97(9 \%)$ \\
\hline - regular small complex tachycardia & $90(9 \%)$ \\
\hline - regular broad complex tachycardia & $1(<1 \%)$ \\
\hline - complete AV block & $6(1 \%)$ \\
\hline uninterpretable & $200(19 \%)$ \\
\hline unreadable (KM) & $100(2 \%)$ \\
\hline atrial fibrillation & $4(4 \%)$ \\
\hline sinus rhythm & $21(21 \%)$ \\
\hline - without ectopy & $18(18 \%)$ \\
\hline - with PACs & $1(1 \%)$ \\
\hline - with PVCs & $2(2 \%)$ \\
\hline other arrhythmias & $4(4 \%)$ \\
\hline - regular small complex tachycardia & $4(4 \%)$ \\
\hline uninterpretable & $71(71 \%)$ \\
\hline
\end{tabular}

tively (upper table); for normal sinus rhythm (without any PACs or PVCs) $0.85,0.83,0.90,0.76$ and 0.84 , (middle table); and for any form of sinus rhythm (with or without PACs or PVCs) 0.80, 0.91, 0.96, 0.65 and 0.83, respectively (lower table).

\section{Discussion}

Hartwacht Arrhythmia is the first remote monitoring program in the Netherlands to use a device (KM) equipped with an algorithm to assess heart rhythm disorders. This study evaluated the ability of the algorithm to categorise 5,982 one-lead ECGs obtained with the KM device, and compared the outcome with the diagnosis provided by a dedicated arrhythmia team. The results show that erroneous classification by the algorithm in the category normal sinus rhythm was rare, assuming sinus rhythm with PACs and/or PVCs to be normal sinus rhythm (PPV 96\%). On the other hand, ECGs classified as possible AF were diagnosed by the arrhythmia team as non-AF in $20 \%$ of cases, and in $81 \%$ of the unclassified ECGs the arrhythmia team was able to provide a diagnosis. These findings show that the KM/algorithm combination in its current form is inadequate for stand-alone clinical evaluation and support the need for additional assessment of the ECGs by an experienced ECG reader.

\section{Atrial fibrillation}

The KM algorithm focusses on detection of AF. In previous studies validating the device, high sensitivity and specificity were obtained for detection of AF. Lau et al. compared the KM ECG to a 12-lead ECG in 109 cardiology patients, a third of whom were in AF, and found a sensitivity of $87 \%$ and specificity of $97 \%$ [1]. Using a similar study design, Haberman compared 381 athletes with healthy volunteers and cardiology clinic patients (sensitivity 94\%, specificity 99\%) [2]. On the other hand, screening of patients for $\mathrm{AF}$ in a geriatric ward resulted in a specificity of $98 \%$ but a disappointing sensitivity of 55\%. The researchers partly attribute these findings to interference/artefacts resulting in poor $P$-wave recognition, which may be an issue in the elderly population.

In the present study, sensitivity and specificity for detection of AF were comparable to previously reported values (92\% and 95\%, respectively). PPV, however, was substantially lower (80\%) and NPV was high (98\%) compared to a previous study using the same algorithm [6]. Most likely, this is attributable to the relatively low prevalence of AF in our study cohort. Of note, comparison of outcomes between the present study and literature should be done with care since all ECGs categorised by the KM algorithm as unclassified or unreadable were often discarded in previous studies, whereas they were included in the present one. Although the majority of AF ECGs are classified correctly, 78 (7\%) are erroneously allocated to the un- 


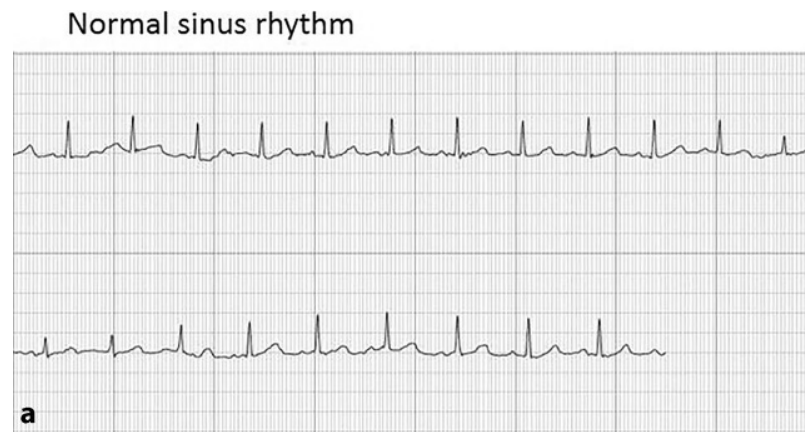

\section{Wide complex tachycardia}

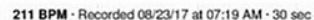

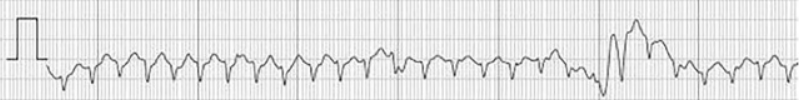

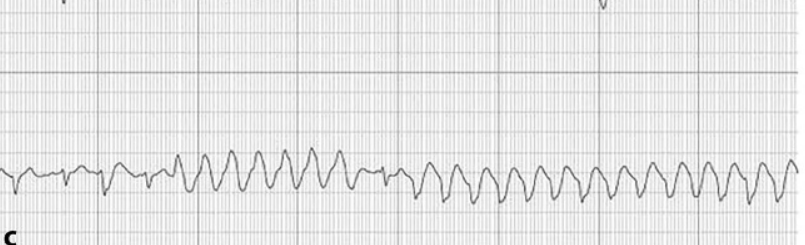

\section{Atrial fibrillation}

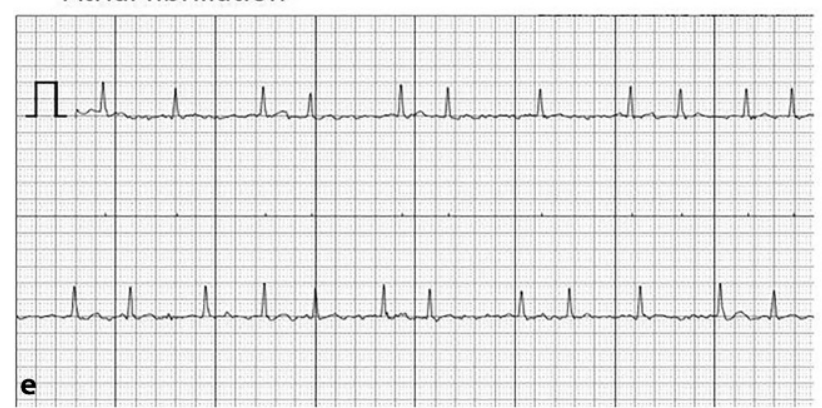

Sinus rhythm with total AV block

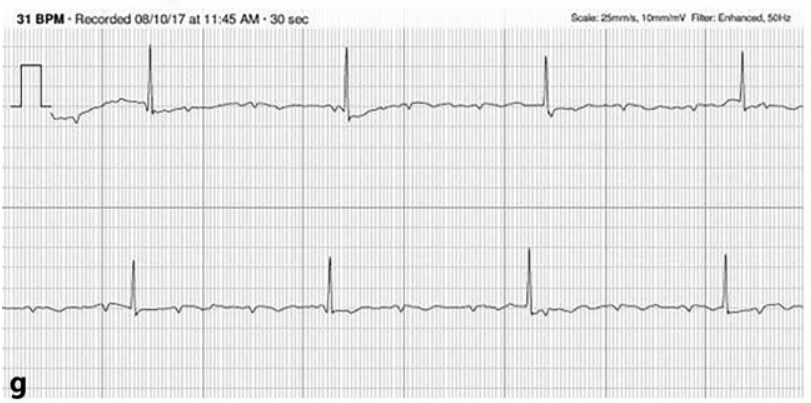

Two fingers of one hand used

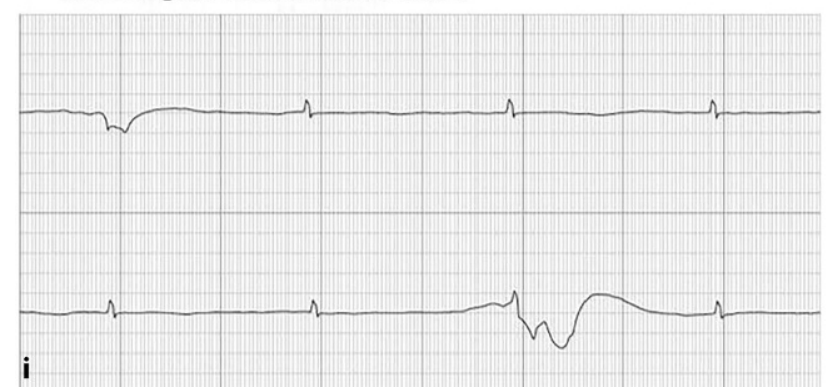

\section{Premature ventricular contraction}

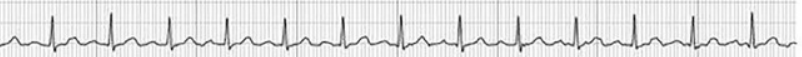

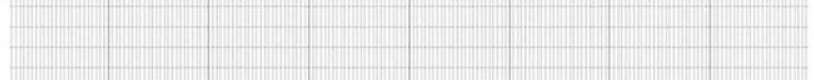

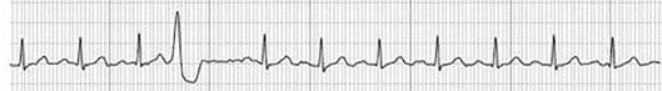

b

Premature atrial contractions

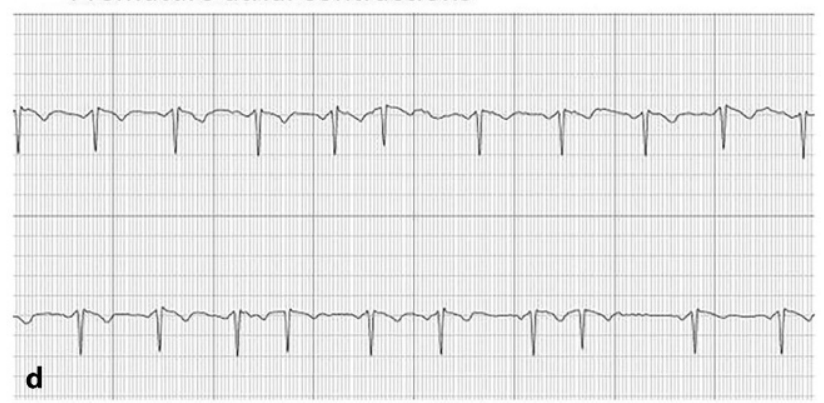

Small complex tachycardia

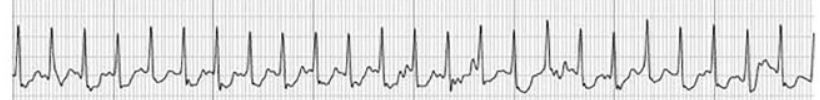

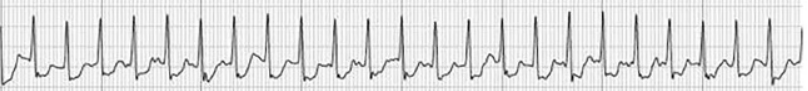
f AliveCor: sinus rhythm, Cardiologist: uninterpretable

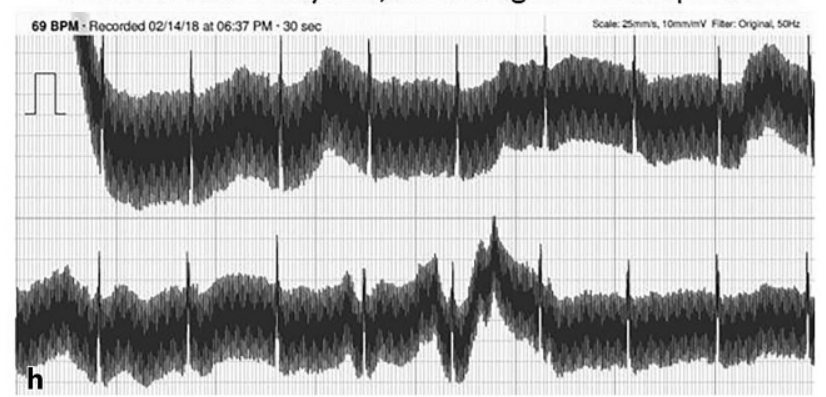

Fig. 4 Various rhythms recorded with the KM device 

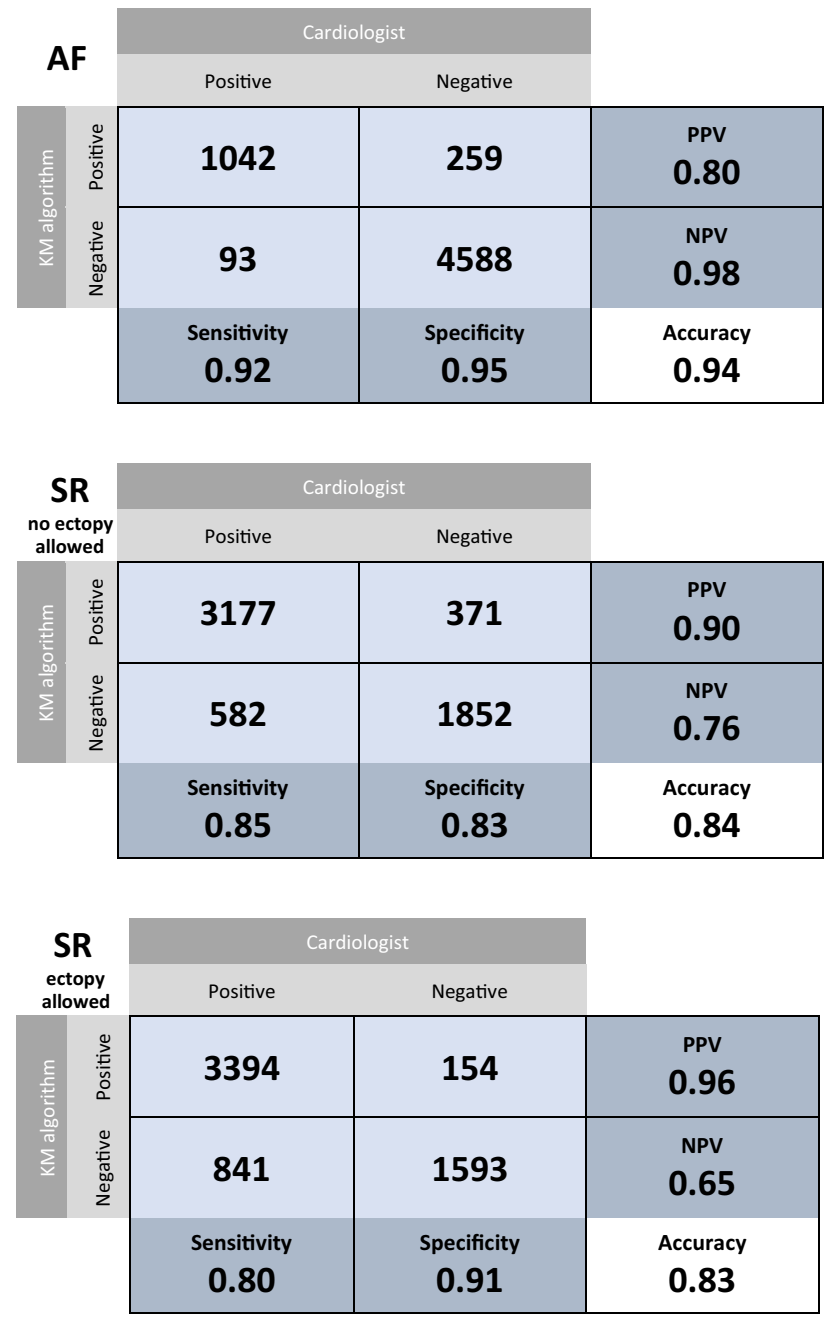

Fig. 5 Two by two matrices of atrial fibrillation (upper) and sinus rhythm without (middle) or with (lower) ectopy

classified category and $11 \mathrm{AF}(<1 \%)$ to the normal sinus rhythm category. In the present study, these ECGs were re-evaluated by the Hartwacht team, which significantly increased diagnostic yield with regard to $\mathrm{AF}$ and shows the added value of the HA program.

The KM device has also been studied as an AF screening instrument in asymptomatic patients aged 65 years or older [7-11], which resulted in $0.8-1.5 \%$ cases of newly diagnosed atrial fibrillation. The present study shows that when using the KM device for screening a general population for $\mathrm{AF}$, two issues need to be considered. Firstly, prevalence of AF will be even lower compared to the present study and consequently PPV will be low with a high number of false positives resulting in a negative impact on costeffectiveness. Secondly, a substantial number of $\mathrm{AF}$ ECGs will be not classified as possible AF emphasising the need for manual assessment of all ECGs.

\section{Ectopy and other non-AF arrhythmias}

Sinus rhythm with ectopy (PACs and/or PVCs) provides a challenge to the algorithm. The algorithm is unable to categorise these arrhythmias properly, as is shown in Fig. 3c, d, where all three KM classes contain a substantial percentage of PACs and PVCs respectively. The importance of recognising these rhythms depends on the indication for participation in HA. Screening for AF would require these rhythms to be discarded whereas for analysis of palpitations of unknown origin, detection of SR with ectopy might provide a useful explanation. This might also apply for other rhythm disorders. Moreover, the algorithm classified 1,033 (17\%) ECGs as unclassifiable. From $81 \%$ of those a diagnosis could be provided by the Hartwacht team, further supporting the need for manual evaluation of all ECGs in such a program.

\section{Limitations}

Several limitations of this study need to be addressed. Firstly, this is a retrospective analysis of a patient population included in the HA program for various reasons and at the discretion of the physician. This may have introduced a substantial selection bias. Consequently, predictive values provided should be interpreted with caution as they vary with prevalence. Furthermore, in the present study the reference for analysis of the 30-second one-lead KM ECGs is the Hartwacht team. In a previous study, Bumgarner et al. reported a specificity of $97 \%$ for detection of AF when comparing the KM classification to interpretation by a cardiologist [6]. However, specificity dropped to $84 \%$ when comparing the KM classification to evaluation of a simultaneously obtained 12-lead ECG. As 12-lead ECGs were not available in the present study, specificity data might have been overestimated.

\section{Conclusion and future perspectives}

The KM device provides a patient-initiated 30-second one-lead ECG of diagnostic quality in ambulatory arrhythmia patients. The present study shows the first remote monitoring arrhythmia program in the Netherlands. Less than $10 \%$ of the ECGs were uninterpretable. For detection of AF, the KM algorithm provides a high NPV, but PPV is relatively low, resulting in the need for manual assessment of all ECGs categorised as other than normal sinus rhythm. However, when the device is used for analysis of arrhythmias of unknown origin, all ECGs should be manually evaluated since non-AF arrhythmias (including ectopy) are poorly recognised by the algorithm and may be classified as normal sinus rhythm. Consequently, for incorporation in a regular healthcare setting a dedicated arrhythmia program is of additional value. The future of remote monitoring for arrhythmias will heavily depend on further enhancement of algorithms, leading 
to improvement of arrhythmia recognition. A practical first step might be the refinement of detection of regular sinus rhythm, which would obviate the need for manual assessment of this category of ECGs.

Conflict of interest J.L. Selder, L. Breukel, S. Blok, A.C. van Rossum, I.I. Tulevski and C.P. Allaart declare that they have no competing interests.

Open Access This article is distributed under the terms of the Creative Commons Attribution 4.0 International License (http://creativecommons.org/licenses/by/4.0/), which permits unrestricted use, distribution, and reproduction in any medium, provided you give appropriate credit to the original author(s) and the source, provide a link to the Creative Commons license, and indicate if changes were made.

\section{References}

1. Lau JK, Lowres N, Neubeck L, et al. Iphone ECG application for community screening to detect silent atrial fibrillation: a novel technology to prevent stroke. Int J Cardiol. 2013;165:193-4.

2. Haberman ZC, Jahn RT, Bose R, et al. Wireless smartphone ECG enables large-scale screening in diverse populations. JCardiovasc Electrophysiol. 2015;26:520-6.

3. Tarakji KG, Wazni OM, Callahan T, et al. Using a novel wireless system for monitoring patients after the atrial fibrillation ablation procedure: the iTransmit study. Heart Rhythm. 2015;12:554-9.
4. Lowres N, Mulcahy G, Gallagher R, et al. Self-monitoring for atrial fibrillation recurrence in the discharge period postcardiac surgery using an iPhone electrocardiogram. Eur J Cardiothorac Surg. 2016;50:44-51.

5. Desteghe L, Raymaekers Z, Lutin M, et al. Performance of handheld electrocardiogram devices to detect atrial fibrillation in a cardiology and geriatric ward setting. Europace. 2017;19:29-39.

6. Bumgarner JM, Lambert CT, Hussein AA, et al. Automated atrial fibrillation detection algorithm using Smartwatch technology. J Am Coll Cardiol. 2018;71:2381-8.

7. Lowres N, Neubeck L, Salkeld G, et al. Feasibility and cost-effectiveness of stroke prevention through community screening for atrial fibrillation using iPhone ECG in pharmacies. The SEARCH-AF study. Thromb Haemost. 2014;111:1167-76.

8. Orchard J, Lowres N, Freedman SB, et al. Screening for atrial fibrillation during influenza vaccinations by primary care nurses using a smartphone electrocardiograph (iECG): A feasibilitystudy. EurJPrevCardiol. 2016;23(2_suppl):13-20.

9. Chan N, Choy C. Screening for atrial fibrillation in 13122 Hong Kong citizens with smartphone electrocardiogram. Heart. 2017;103:24-31.

10. Halcox JPJ, Wareham K, Cardew A, et al. Assessment of remote heart rhythm sampling using the aliveCor heart monitor to screen for atrial fibrillation: the REHEARSE-AF study. Circulation. 2017;136:1784-94.

11. Evans GF, Shirk A, Muturi P, Soliman EZ. Feasibility of using mobileECGrecordingtechnology to detectatrialfibrillation in low-resource settings. Glob Heart. 2017;12:285-9. 